\title{
Mindfulness, empatía y compasión: Evolución de la empatía a la compasión en el ámbito sanitario
}

\author{
Miguel Bellosta-Batalla ${ }^{1}$, Elena Garrote-Caparrós ${ }^{2}$, Dra. Josefa Pérez-Blasco ${ }^{3}$, Dr. Luis Moya- \\ Albiol ${ }^{4}$ y Dr. Ausiàs Cebolla ${ }^{5, *}$ \\ 1 Departamento de Psicobiología, Universitat de València; miguel.bellosta@uv.es \\ 2 Departamento de Psicobiología, Universitat de València; elena.garrote.caparros@gmail.com \\ 3 Departamento de Psicología Evolutiva y de la Educación, Universitat de València; josefa.perez@uv.es \\ 4 Departamento de Psicobiología, Universitat de València; luis.moya@uv.es \\ 5 Departamento de Personalidad, Evaluación y Tratamientos Psicológicos. Universitat de València \\ * Autor correspondencia: ausias.cebolla@uv.es
}

Recibido: 14/06/2018; Aceptado: 27/08/2018; Publicado: 30/01/2019

Resumen: En el ámbito de la atención a la salud mental, la empatía es un aspecto especialmente importante, ya que supone la base sobre la que se sostiene el vínculo terapéutico y se articulan las diferentes actuaciones psicológicas, al facilitar un entendimiento de la vida y de las situaciones de los pacientes. En este sentido, las intervenciones basadas en mindfulness y compasión (IBMC) se han mostrado efectivas para aumentar la empatía en los profesionales sanitarios. Sin embargo, actualmente siguen existiendo algunas inconsistencias en el estudio de la empatía y su relación con mindfulness y compasión. En este artículo, se expone una visión global de estos constructos, subrayando la importancia de identificar los sesgos en la inferencia sobre el significado de las vivencias ajenas, y analizando el impacto de mindfulness y compasión en la gestión saludable de las emociones y su implicación en el acercamiento al sufrimiento de los demás. Finalmente, se introduce un modelo explicativo en el que se integra lo anterior, y se sugiere la influencia de mindfulness en la evolución de la empatía a la compasión. El estudio de esta asociación supone un aspecto fundamental en la investigación acerca de los beneficios de las IBMC sobre la empatía en el ámbito sanitario.

Palabras Clave: Empatía; Mindfulness; Compasión; Psicoterapia; Psicología Clínica y de la Salud; Sesgos en la Inferencia.

Abstract: In the area of attention to mental health, empathy is an especially important aspect, since it is the basis on which the therapeutic link is maintained and the different psychological actions are articulated, facilitating an understanding of life and patient's situations. In this regard, mindfulness and compassion-based interventions (MCBI) have shown to be effective in increasing empathy in healthcare professionals. However, there are still some inconsistencies in the study of empathy and its relation with mindfulness and compassion. In this article, a global vision of these constructs is exposed, stressing the importance of identifying the biases in the inference about the meaning of the experiences of others, and analyzing the impact of mindfulness and compassion in the healthy management of emotions and its involvement in the approach to the other's suffering. Finally, an explanatory model is introduced in which the above is integrated, and the influence of mindfulness in the evolution of empathy to compassion is suggested. The study of this association supposes a fundamental aspect in the investigation about the benefits of the MCBI on empathy in the healthcare field.

Key words: Empathy; Mindfulness; Compassion; Psychotherapy; Clinical and Health Psychology; Biases in the Inference. 


\section{Introducción}

La empatía es un aspecto esencial en el entorno sanitario, ya que supone uno de los elementos básicos en los que se sostiene el vínculo terapéutico y sobre el que se articula la relación de ayuda [1,2]. En el ámbito de la atención a la salud mental, esta habilidad adquiere una importancia fundamental, al encontrarse a la base del entendimiento de las experiencias de los pacientes, y guiar así las diferentes estrategias utilizadas en la búsqueda de su bienestar [3,4]. Esto último ha elevado el interés en el estudio y el entrenamiento de la empatía en la formación universitaria de los psicólogos clínicos y sanitarios [5].

En relación con lo anterior, las intervenciones basadas en mindfulness y compasión (IBMC) se han establecido como una alternativa útil para mejorar la empatía en los profesionales sanitarios [6,7]. Estas intervenciones ayudan a desarrollar una serie de actitudes y habilidades estrechamente relacionadas con la empatía, y que ejercen un efecto favorable sobre el vínculo terapéutico [8-10]. Se ha visto que el entrenamiento en mindfulness en psicoterapeutas influye beneficiosamente en la salud y en la evolución sintomática de sus pacientes, sin necesidad de que su aprendizaje se incluya de forma explícita en las sesiones [11]. Siguiendo esta línea, algunos autores señalan que la práctica de mindfulness implica la adopción de una actitud de curiosidad, apertura, aceptación y amabilidad [12], aspectos esenciales en el ejercicio de la psicoterapia, y que en última instancia, podrían facilitar una escucha sincera y el establecimiento de un vínculo terapéutico eficaz con los pacientes [13,14]. Además, la compasión -un sentimiento saludable que se genera ante la vivencia del sufrimiento, y en el que se incluye la intención de aliviarlo- puede ser entendida como la base en la que se sostienen las diferentes actuaciones psicológicas, al guiar una búsqueda del bienestar en los demás [15]. Sin embargo, actualmente siguen existiendo algunas inconsistencias en este ámbito de investigación, y especialmente, en lo que se refiere al entendimiento de la empatía y su relación con la compasión, así como a los efectos de mindfulness sobre estos constructos en el ámbito sanitario.

En este artículo, se aborda el estudio de la empatía y de aquellos fenómenos que pueden influir negativamente en ella, y que impiden una adecuada valoración del estado interno de los pacientes. Así mismo, se exponen los aspectos socioemocionales vinculados a mindfulness y compasión, y se analizan sus semejanzas con la empatía. Finalmente, se sugiere un modelo explicativo en el que se establece la asociación entre estos constructos, incidiendo en la evolución de la empatía a la compasión, y señalando las ventajas de introducir las IBMC en la formación universitaria de los psicólogos clínicos y sanitarios.

\section{El estudio de la empatía y su significado en el ámbito sanitario}

En las investigaciones actuales sobre la empatía, ésta es entendida como una habilidad en la que se integran una serie de aspectos cognitivos y emocionales, siendo un elemento básico en el acercamiento a las experiencias de aquellos que se encuentran a nuestro alrededor [16,17]. La empatía cognitiva consiste en la habilidad de inferir el estado cognitivo y emocional de un individuo, basándose en la adopción de su perspectiva. A su vez, la empatía emocional supone una sintonización afectiva con ese estado, que se genera gracias a un acercamiento a su situación, y que sirve de guía en la elaboración intelectual que se realiza sobre él. En esta aproximación, es necesario que se establezca una separación entre las emociones ajenas y las de uno mismo, evitando así una respuesta de simpatía $[18,19]$. Esta última implica una fusión afectiva en la que el observador hace suyo el estado emocional de los demás, y acaba sumergiéndose en él sin que exista un verdadero entendimiento [17]. En este sentido, la gestión saludable de las emociones es especialmente importante, ya que la simpatía suele evolucionar en sentimientos de ansiedad ante la vivencia del sufrimiento ajeno, siendo ésta una reacción que se conoce como angustia empática [20].

Los aspectos cognitivos y emocionales de la empatía están íntimamente relacionados entre sí, ya que su establecimiento implica una asociación entre ambos y el ambiente social en el que se desenvuelve el individuo [21]. Además, los estudios indican que aunque estos componentes estarían regulados por sistemas neuronales diferentes, no funcionan de forma aislada, sino que lo hacen en continua interacción [17]. La adopción de perspectiva nos ayuda a inhibir la visión egocéntrica que se activa de forma automática en la apreciación de nuestras experiencias, evaluando las situaciones 
específicas a las que se enfrentan los demás [22,23]. Esto último supone un elevado esfuerzo cognitivo y atencional, y es esencial en la valoración sobre su estado emocional [24]. Sin embargo, es importante señalar que en la empatía no existe una similitud exacta en relación con las vivencias ajenas, al encontrarnos ante un fenómeno subjetivo en el que intentamos inferir el significado de lo que le sucede a los que están a nuestro alrededor [19]. Según algunos autores, una vez que se ha entendido lo que está viviendo un individuo, es necesario volver a una visión egocéntrica para que se produzca la empatía emocional, imaginando lo que sentiríamos nosotros si estuviésemos en esa situación, y evocando una serie de emociones asociadas a ella [25]. No obstante, si bien estas emociones van a ser acordes a la escena imaginada, no siempre serán similares a las suyas. Es posible que existan algunos errores en la interpretación acerca del impacto que esta situación adquiere en los demás, al evaluarla sobre la base de nuestros esquemas cognitivos, valores y aprendizajes [26].

Estos errores en la interpretación se han denominado sesgos en la inferencia [27], y suelen influir negativamente en la empatía cognitiva y afectar a las emociones del observador. En este sentido, algunos estudios señalan que la adopción de perspectiva no siempre implica una exactitud en la inferencia acerca del estado cognitivo y emocional ajeno, y que algunas veces, supone incluso una importante fuente de error, existiendo una falsa ilusión de sintonía [28]. Esto es debido a que en este proceso el observador simplemente accede a la información de la que dispone -sesgada o no- sobre la situación que está viviendo un individuo, integrándola en su valoración acerca de sus experiencias. Además, se ha visto que el acercamiento egocéntrico al sufrimiento está asociado a una afectividad negativa [29], ya que en el intento de entender las vivencias ajenas, nos imaginamos a nosotros mismos en una situación similar. Esto último facilita la adopción de una serie de sesgos en la inferencia, evocando unas emociones que no necesariamente se asemejan a las que está sintiendo el individuo [19], y que en última instancia, pueden generar una respuesta de simpatía y/o de angustia empática. Es por ello por lo que algunos autores acentúan la importancia de explorar las experiencias y el sistema de significados de aquellos que se encuentran a nuestro alrededor para que la adopción de su perspectiva sea verdaderamente útil y favorezca la empatía, alcanzando así un elevado entendimiento de sus vivencias [28].

Siguiendo esta línea, en el ámbito sanitario la empatía se ha entendido como una actividad en la que se realiza una exploración y un acompañamiento en las situaciones vitales de los pacientes, y en la que se incluye la intención de aliviar su sufrimiento [30,31]. En este enfoque, se amplía la visión anterior de la empatía, al integrar una serie de aspectos interpersonales que ayudan a generar un espacio de seguridad, y que se encuentran asociados a la evolución saludable de los pacientes. Así, se ha señalado la importancia de los siguientes elementos relacionados con la empatía: la aceptación incondicional, en la que se afirma el valor del individuo independientemente de sus actos y sentimientos, la adopción de una actitud amable, y la autenticidad en el vínculo establecido, identificando y elaborando el efecto que adquiere en uno mismo [32,33]. Vemos cómo esta definición de empatía se asemeja bastante a lo que actualmente se entiende por compasión, al integrar una actitud amable y sentimientos saludables hacia las experiencias de los demás, en los que se incluye una búsqueda de alivio de su sufrimiento. Este aspecto ha sido subrayado en algunos estudios, indicando que el vínculo empático no sólo supone un entendimiento de las situaciones ajenas, sino que implica además un ajuste de la sensibilidad y de las respuestas del psicoterapeuta a las necesidades y vivencias del paciente, adaptándose a la naturaleza evolutiva de esta situación interpersonal, y buscando de forma activa un aumento en su bienestar [34,35].

Sin embargo, el hecho que en el ámbito académico se hayan ignorado estas variables ha supuesto que algunos autores señalen el uso egoísta que a veces se puede realizar de la empatía, aprovechándose para incrementar el beneficio individual [26,36], fundamentalmente si existe una elevada empatía cognitiva y una baja empatía emocional [17]. Esto último ha servido para iniciar un interesante diálogo acerca de las semejanzas entre empatía y compasión, así como sobre los aspectos en los que estos constructos se diferencian y enfrentan incluso en su significado [37]. No obstante, y en vez de asumir que estas diferencias suponen un alejamiento de los esfuerzos de investigación, los avances en el estudio de la empatía -y su visión como un fenómeno exclusivamente cognitivo y 
emocional- nos pueden ayudar a explorar los aspectos involucrados en su evolución a la compasión, y analizar la influencia beneficiosa de mindfulness en este sentido.

\section{Mindfulness y compasión: una visión socioemocional}

Las intervenciones basadas en mindfulness (IBM) se han introducido con éxito en el entorno sanitario, extendiéndose en nuestra sociedad gracias a sus beneficios sobre la salud y el bienestar [3840]. En un inicio, el estudio de estas intervenciones estuvo enfocado en sus efectos sobre el estrés y la prevención de recaídas en depresión [41-43], si bien actualmente ha aumentado el interés acerca de su influencia en una serie de actitudes relacionadas con el establecimiento de las vinculaciones afectivas, siendo la empatía una de las variables más investigadas [44,45]. La inclusión de los aspectos socioemocionales en el estudio de mindfulness se aprecia así mismo en la evolución que ha experimentado este constructo en el ámbito académico.

En una de las primeras definiciones que aparecieron sobre mindfulness, éste es entendido como un estado mental en el que se atiende a lo que sucede en nosotros mismos y a nuestro alrededor con una actitud de aceptación y ausencia de juicios [41]. Se acentúa así la importancia de un acercamiento no sesgado a la experiencia, en el que se intenta inhibir la influencia de nuestros aprendizajes en la interpretación de las situaciones a las que nos enfrentamos. Además, en esta definición se incluye la aceptación como un aspecto fundamental, y que se encuentra relacionado con la gestión saludable del estrés y la mejora de la salud del individuo [46]. Algunos autores han señalado los diferentes mecanismos que subyacen a los efectos beneficiosos de mindfulness, agrupándolos en los siguientes: la regulación de la atención, el aumento de la sensibilidad interoceptiva, la gestión eficaz de las emociones (en la que se incluye la exposición y revalorización), y la variación en el auto-concepto, al ser abordado con una mayor flexibilidad $[47,48]$. Estos aspectos están vinculados entre sí, ya que la atención a nuestras experiencias somáticas y a las elaboraciones verbales realizadas sobre ellas -unida a una actitud de apertura y aceptación- favorece un aumento de la autoconsciencia y una gestión eficaz de las emociones, ayudándonos a relacionarnos de forma saludable con nuestras vivencias.

Sin embargo, esta definición de mindfulness se ha ido ampliando al integrar diferentes elementos actitudinales asociados a las vinculaciones afectivas, alejándose de su conceptualización como un simple fenómeno cognitivo en el que se encuentra involucrada la atención [49]. Siguiendo esta línea, son varios los autores que subrayan la importancia de la ética y la actitud con la que el individuo se relaciona con sus experiencias y los que están a su alrededor $[13,50]$, señalando que la amabilidad es un aspecto fundamental del significado y la esencia de mindfulness [12]. La práctica de mindfulness implica una acogida saludable del sufrimiento, suponiendo así una forma implícita de autocompasión [51]. Además, se ha visto que las IBM influyen beneficiosamente en la empatía y la compasión, sin necesidad de que éstas sean incluidas en las sesiones de la intervención [51,52]. Esto último indica que el entrenamiento en mindfulness favorece el desarrollo de un conjunto de actitudes y habilidades básicas que se encuentran íntimamente relacionadas con la compasión. Así mismo, en los últimos años ha aumentado el número de intervenciones basadas en compasión (IBC) en las que se aborda su entrenamiento de forma explícita [53], y son varias las IBM en las que se introducen elementos específicos de las IBC [54,55].

En relación con lo anterior, en la compasión nos encontramos con diferentes aspectos cognitivos y emocionales, unidos a la intención y a la motivación de aliviar el sufrimiento [56]. En este sentido, la compasión incluye la identificación de las fuentes del sufrimiento, la adopción de una actitud amable en este acercamiento, el aprendizaje de que su vivencia -y la búsqueda de un estado de bienestar- es una experiencia humana universal, la sintonía afectiva con ese estado, la gestión eficaz y la separación de las emociones negativas asociadas a él, y una serie de sentimientos saludables en los que se incluye la intención de aliviarlo, y que guían las acciones enfocadas a ello $[57,58]$. Esta visión integra varios aspectos esenciales de la empatía, y supone una ampliación de este constructo. Así mismo, en ella se acentúa la importancia de la regulación de los estados internos y la separación de las emociones ajenas, algo fundamental en el establecimiento de un vínculo saludable y eficaz en el ámbito sanitario [14,59]. 


\section{La evolución de la empatía a la compasión}

Sintetizando lo anterior, en el establecimiento de una empatía saludable -y que suponga un acercamiento efectivo a las situaciones de los demás- es fundamental que exista una separación entre las experiencias ajenas y las de uno mismo [60]. En la vertiente cognitiva, los sesgos en la inferencia pueden estar introduciendo diferentes errores en la empatía, alcanzando así una falsa ilusión de sintonía [28]. Esto último afectaría de forma negativa al vínculo establecido, impidiendo que el individuo sienta que está siendo realmente entendido [3]. Es por ello por lo que es necesario superar esa visión egocéntrica en la que interpretamos las situaciones ajenas basándonos automáticamente en nuestras experiencias [22,23], y explorar el significado que adquieren en los demás en función de su sistema de valores y aprendizajes [26]. En la vertiente emocional, esta separación es esencial para evitar que nuestras emociones actuales influyan en la apreciación sobre el estado afectivo de aquellos que se encuentran a nuestro alrededor [60]. Así mismo, es necesaria una gestión eficaz de las emociones asociadas a la empatía, inhibiendo una respuesta de simpatía y/o de angustia empática $[18,20]$. Siguiendo esta línea, es fundamental el establecimiento de una base segura y un equilibrio emocional que nos ayude a sostener el sufrimiento sin vernos sumergidos en él [61], acogiendo las situaciones ajenas de forma saludable.

Si bien los aspectos señalados hasta ahora facilitan el establecimiento de una escucha sincera en la que se entiendan realmente las experiencias de los demás, y en la que no exista una reacción negativa ante su sufrimiento-, esto no garantiza que el vínculo empático vaya a ser beneficioso para ellos $[19,26,36]$. La evolución de la empatía a la compasión supone la adopción de una actitud amable en este acercamiento empático, y se acompaña siempre de la intención de aliviar el sufrimiento [5658]. Además, en la compasión nos encontramos con una importante variación en la experiencia interna del observador. Así, en la empatía emocional existe una sintonización afectiva con las situaciones ajenas, de forma que la emoción que surge en él es similar o se acerca a lo que está viviendo el individuo, ayudándole en la elaboración intelectual sobre su estado actual. Sin embargo, en su evolución a la compasión se generan además una serie de emociones saludables en las que se incluye la intención de aliviar su sufrimiento, y que finalmente, favorecen la aparición de la acción compasiva [57,62]. En la figura 1, se introduce un modelo explicativo sobre la empatía en el que se expone su evolución a la compasión, y en el que se aprecia la influencia negativa de la simpatía y de los sesgos en la inferencia.

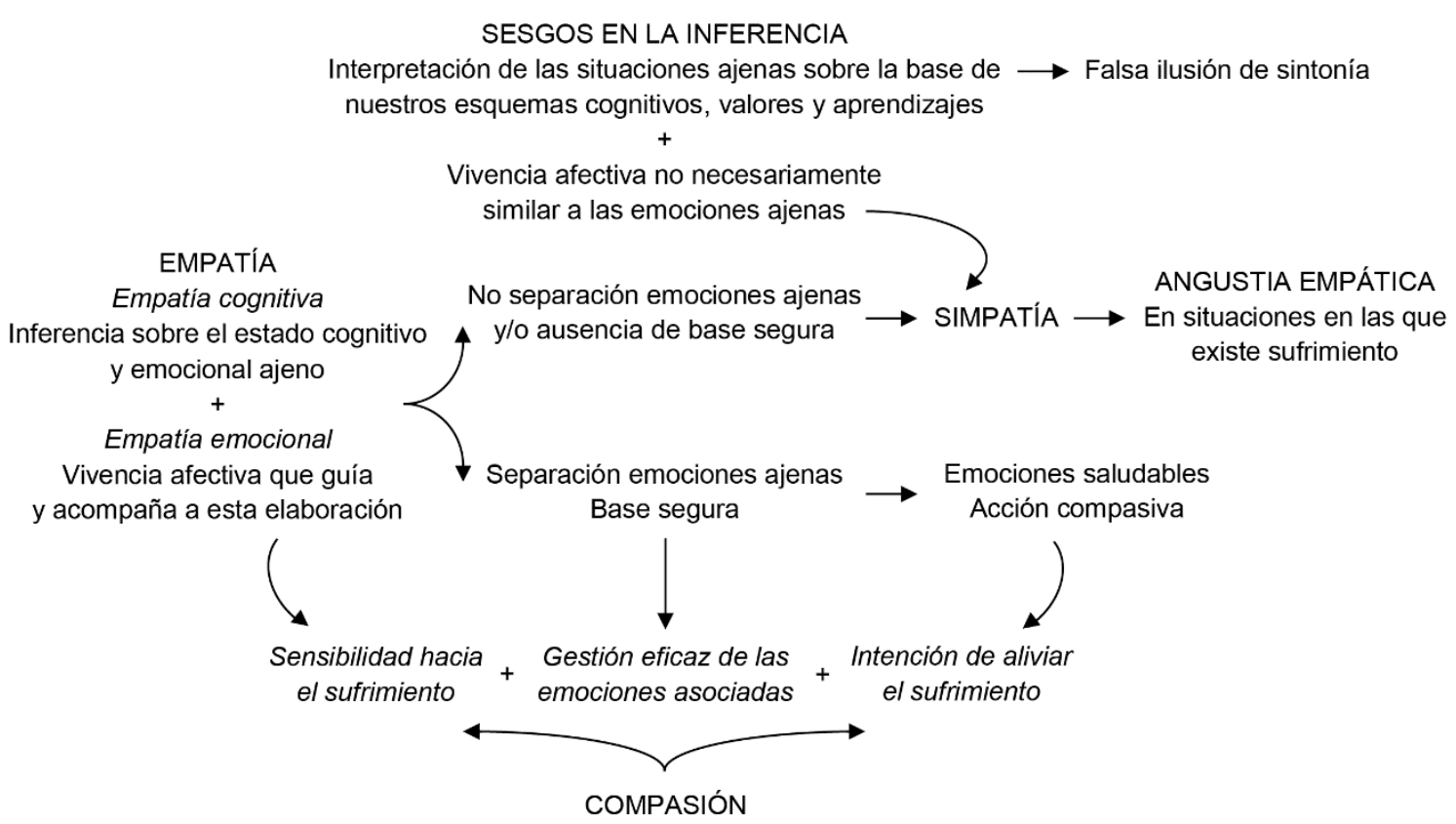

Figura 1 Análisis de la evolución de la empatía a la compasión, y de la influencia negativa de la simpatía y de los sesgos en la inferencia. 
En relación con lo anterior, vemos cómo en el establecimiento de una empatía saludable, y en la intención de aliviar el sufrimiento de los demás, se integran los diferentes componentes de la compasión: el aumento de la sensibilidad hacia el sufrimiento ajeno (empatía cognitiva y emocional), la gestión eficaz de las emociones asociadas a él (evitando una respuesta de simpatía y/o de angustia empática), y las acciones que se realizan con la intención de aliviarlo [57,58]. Así, el ejercicio de la psicoterapia puede ser entendido como un acto compasivo en sí mismo, en el que se intenta entender la situación vital de un individuo y se le acompaña en la búsqueda de alivio de su sufrimiento, ayudándole a alcanzar un estado de bienestar sostenible [15,63]. Es por ello por lo que la compasión constituye el eje vertebrador en el que se sostienen las diferentes actuaciones psicológicas, independientemente del enfoque y la escuela desde la que se intervenga. En este sentido, son varios los autores que sugieren las ventajas de introducir las IBMC en la formación universitaria de los psicólogos clínicos y sanitarios $[8,9,64,65]$, señalando sus beneficiosos en la empatía y su evolución a la compasión.

\section{Mindfulness, empatía y compasión en el ámbito sanitario}

En los últimos años, ha aumentado el volumen de investigaciones acerca de los efectos de las IBMC sobre la empatía en los profesionales sanitarios [6,7]. Estas intervenciones ayudan a desarrollar una serie de habilidades íntimamente relacionadas con la empatía, y que en última instancia, favorecen su evolución a la compasión. Siguiendo esta línea, y atendiendo a los aspectos asociados a la práctica de mindfulness y compasión [47,57], vemos cómo ambos constructos adquieren una gran importancia en el establecimiento de una empatía saludable, al inhibir la influencia negativa de los sesgos en la inferencia y evitar una respuesta de simpatía y/o de angustia empática al enfrentarnos al sufrimiento ajeno.

La regulación de la atención está implicada en la adopción y el entendimiento de la perspectiva de los demás [25,28], y facilita una escucha amplia en la que se atiende al significado que subyace a las experiencias de los pacientes [66]. En este acercamiento, el aumento de la autoconsciencia es especialmente necesario, siendo uno de los elementos básicos en la formación de los psicólogos clínicos y sanitarios [67]. Esto es debido a que un elevado autoconocimiento favorece la separación entre las vivencias ajenas y las de uno mismo, algo fundamental en el establecimiento de la empatía [60]. En relación con lo anterior, la atención a la experiencia en su estado actual nos ayuda a inhibir la influencia de nuestros aprendizajes en la interpretación de las situaciones a las que nos enfrentamos [41], identificando los sesgos en la inferencia sobre el estado cognitivo y emocional de los demás [26,27]. Esto último, unido a la exploración de su sistema de significados, facilita el entendimiento de sus vivencias [28], influyendo beneficiosamente sobre la sensación de sintonía y la efectividad del vínculo terapéutico [3].

En este proceso, la gestión eficaz de las emociones supone un aspecto esencial, al fomentar un equilibrio emocional y evitar una fusión afectiva con el estado interno de los pacientes [18,20]. Además, la actitud autocompasiva y las acciones de autocuidado son fundamentales en la acogida de su sufrimiento, ya que nos ayudan a generar una base segura sobre la que es posible sostenerlo sin vernos sumergidos en él $[61,68]$. Así mismo, la sensibilidad interoceptiva adquiere una gran importancia, al asociarse a un aumento en la exposición y revalorización de la emoción [69]. Estas estrategias se encuentran involucradas en la separación entre nuestras experiencias y las situaciones de los demás, así como en la sintonización afectiva que acompaña a la empatía emocional [70].

Basándonos en los estudios anteriores, es posible afirmar que existe una importante asociación entre mindfulness, empatía y compasión. En el ámbito sanitario, estos constructos influyen en el desarrollo de una serie de habilidades estrechamente relacionadas con el establecimiento de un vínculo saludable y eficaz con los pacientes $[10,13,14]$. En este sentido, los beneficios de mindfulness en la evolución de la empatía a la compasión sugieren las ventajas de introducir las IBMC en la formación universitaria de los psicólogos clínicos y sanitarios [8,9,65]. 


\title{
6. Conclusiones
}

La empatía es una habilidad esencial en el entendimiento de las situaciones vitales de aquellos que se encuentran a nuestro alrededor, siendo uno de los aspectos más importantes en el ámbito de la psicología clínica y de la salud, ya que supone la base sobre la que se articulan las diferentes actuaciones psicológicas [3,5]. En los últimos años, ha aumentado el número de investigaciones en las que se señala la influencia de las IBMC sobre la empatía [6,7], sugiriendo las ventajas de incluir estas intervenciones en la formación universitaria de los psicólogos clínicos y sanitarios [8,9,65]. Sin embargo, el establecimiento de una empatía saludable no implica que esta habilidad vaya a ser utilizada en beneficio de los demás [26,36]. Es por ello por lo que actualmente siguen existiendo algunas inconsistencias en el estudio de la empatía, y especialmente, en lo que se refiere a su relación con la compasión.

En este artículo, se ha introducido un modelo explicativo en el que se expone la asociación entre mindfulness, empatía y compasión en el ámbito sanitario, señalando los beneficios de mindfulness en el establecimiento de los vínculos saludables. En él se acentúa la importancia de la separación entre las experiencias ajenas y las de uno mismo, inhibiendo los sesgos en la inferencia sobre el estado cognitivo y emocional de los demás. Siguiendo esta línea, es fundamental que en la interpretación de las vivencias de los pacientes adoptemos un acercamiento del estilo "si yo fuese tú", en vez de "si yo estuviese en tu situación" [71]. La regulación de la atención y el aumento de la autoconsciencia asociados a la práctica de mindfulness son esenciales para evitar estos errores en la apreciación acerca de su estado interno [41], incrementando su sensación de que están siendo entendidos, y ejerciendo un efecto beneficioso sobre el vínculo terapéutico [3]. Así mismo, es importante que en la aproximación a las emociones de los demás no se produzca una fusión afectiva con su sufrimiento, evitando así una respuesta de simpatía y/o de angustia empática [18,20]. La gestión eficaz de las emociones y la existencia de una base segura son fundamentales en este sentido [61], al aumentar nuestra habilidad para "surfear" ese estado emocional, en vez de vernos sumergirnos en él [72]. Finalmente, la actitud amable hacia la experiencia y la intención de aliviar el sufrimiento ajeno son elementos necesarios en la evolución de la empatía a la compasión [56,57], y facilitan el establecimiento de un vínculo saludable con los pacientes [10,13,68].

El estudio de la asociación entre mindfulness, empatía y compasión supone un aspecto especialmente importante en la investigación acerca de los efectos de las IBMC en el ámbito sanitario. Estas intervenciones se anuncian como una estrategia de gran utilidad en la formación universitaria de los psicólogos clínicos y sanitarios, influyendo beneficiosamente sobre la habilidad de escuchar, sostener, acompañar y aliviar el sufrimiento, y siendo esenciales en la evolución de la empatía a la compasión.

Agradecimientos: Este estudio ha sido posible gracias a la financiación de las Ayudas a la Investigación 2017 de la Fundación Banco Sabadell, y las Ayudas para la formación de personal investigador predoctoral de la Generalitat Valenciana (ACIF/2016/383) y del Fondo Social Europeo.

Conflictos de Intereses: Los autores no declaran conflicto de intereses.

\author{
Abreviaturas \\ Las siguientes abreviaturas son usadas en este manuscrito: \\ IBMC: Intervenciones basadas en mindfulness y compasión \\ IBM: Intervenciones basadas en mindfulness \\ IBC: Intervenciones basadas en compasión
}




\section{Referencias Bibliográficas}

1. Keijsers GPJ, Schaap CPDR, Hoogduin CAL. The impact of interpersonal patient and therapist behavior on outcome in cognitive-behavior therapy. Behav Modif. 2000; 24(2): 264-297. http://dx.doi.org/10.1177/0145445500242006

2. Lambert MJ, Barley DE. Research summary on the therapeutic relationship and psychotherapy outcome. Psychotherapy (Chic). 2001; 38(4): 357-361. http://dx.doi.org/10.1037/0033-3204.38.4.357

3. Elliott R, Bohart AC, Watson JC, Greenberg LS. Empathy. Psychotherapy (Chic). 2011; 48(1): 43-49. http://dx.doi.org/10.1037/a0022187

4. Norcross JC, Wampold BE. Evidence-based therapy relationships: Research conclusions and clinical practices. Psychotherapy (Chic). 2011; 48(1): 98-102. http://dx.doi.org/10.1037/a0022161

5. Norcross JC, editor. Psychotherapy relationships that work. 2nd. ed. New York (NY): Oxford University Press; 2011.

6. Lamothe M, Rondeau É, Malboeuf-Hurtubise C, Duval M, Sultan S. Outcomes of MBSR or MBSR-based interventions in health care providers: A systematic review with a focus on empathy and emotional competencies. Complement Ther Med. 2016; 24: 19-28.

http://dx.doi.org/10.1016/j.ctim.2015.11.001

7. Bibeau M, Dionne F, Leblanc J. Can compassion meditation contribute to the development of psychotherapists' empathy? A review. Mindfulness. 2016; 7(1): 255-263.

http://dx.doi.org/10.1007/s12671-015-0439-y

8. Bruce N, Shapiro SL, Constantino MJ, Manber R. Psychotherapist mindfulness and the psychotherapy process. Psychotherapy (Chic). 2010; 47(1): 83-97.

http://dx.doi.org/10.1037/a0018842

9. Davis DM, Hayes JA. What are the benefits of mindfulness? A practice review of psychotherapy-related research. Psychotherapy (Chic). 2011; 48(2): 198-208.

http://dx.doi.org/10.1037/a0022062

10. Keane A. The influence of therapist mindfulness practice on psychotherapeutic work: A mixed-methods study. Mindfulness. 2014; 5(6): 689-703.

http://dx.doi.org/10.1007/s12671-013-0223-9

11. Grepmair L, Mitterlehner F, Loew T, Bachler E, Rother W, Nickel M. Promoting mindfulness in psychotherapists in training influences the treatment results of their patients: A randomized, double-blind, controlled study. Psychother Psychosom. 2007; 76(7): 332-338.

http://dx.doi.org/10.1159/000107560

12. Siegel DJ. The mindful brain: Reflection and attunement in the cultivation of well-being. New York (NY): W.W. Norton \& Company; 2007.

13. Brito G. Rethinking mindfulness in the therapeutic relationship. Mindfulness. 2014; 5(4): 351-359. http://dx.doi.org/10.1007/s12671-012-0186-2

14. Leonard HD, Campbell K, Gonzalez VM. The relationships among clinician self-report of empathy, mindfulness, and therapeutic alliance. Mindfulness. 2018; 9(6): 1837-1844. http://dx.doi.org/10.1007/s12671-018-0926-z.

15. Gilbert $\mathrm{P}$, editor. Compassion: Conceptualisations, research and use in psychotherapy. New York (NY): Routledge; 2005.

16. Singer T, Lamm C. The social neuroscience of empathy. Ann N Y Acad Sci. 2009; 1156(1): 81-96. http://dx.doi.org/10.1111/j.1749-6632.2009.04418.x

17. Moya-Albiol L. La empatía. Barcelona: Plataforma Actual; 2018.

18. Decety J, Lamm C. Empathy versus personal distress. Recent evidence from social neuroscience. En: Decety J, Ickes W, editores. The social neuroscience of empathy. Cambridge: MIT Press; 2009. p. 199-213.

19. Zahavi D, Rochat P. Empathy $\neq$ sharing: Perspectives from phenomenology and developmental psychology. Conscious Cogn. 2015; 36: 543-553. http://dx.doi.org/10.1016/j.concog.2015.05.008 
20. Klimecki O, Singer T. Empathic distress fatigue rather than compassion fatigue? Integrating findings from empathy research in psychology and social neuroscience. En: Oakley B, Knafo A, Madhavan G, Wilson DS, editores. Pathological altruism. New York (NY): Oxford University Press; 2012. p. 368-383.

21. Davis MH. Empathy: A social psychological approach. Boulder (CO): Westview Press; 1996.

22. Royzman EB, Cassidy KW, Baron J. "I know, you know”: Epistemic egocentrism in children and adults. Rev Gen Psychol. 2003; 7(1): 38-65. http://dx.doi.org/10.1037/1089-2680.7.1.38

23. Epley N, Keysar B, Van Boven L, Gilovich T. Perspective taking as egocentric anchoring and adjustment. J Pers Soc Psychol. 2004; 87(3): 327-339. http://dx.doi.org/10.1037/0022-3514.87.3.327

24. Decety J. Perspective taking as the royal avenue to empathy. En: Malle BF, Hodges SD, editores. Other minds: How humans bridge the divide between self and others. New York (NY): Guilford Press; 2005. p. 143-157.

25. Chiu CD, Yeh YY. In your shoes or mine? Shifting from other to self perspective is vital for emotional empathy. Emotion. 2018; 18(1): 39-45. http://dx.doi.org/10.1037/emo0000346

26. Breithaupt F. Kulturen der empathie. Frankfurt am Main: Suhrkamp; 2009.

27. Bellosta-Batalla M, Cebolla A, Pérez-Blasco J, Moya-Albiol L. La empatía en el ejercicio y formación de los psicólogos clínicos y sanitarios, y su relación con mindfulness y compasión. Rev Argent Clin Psic. En prensa 2018.

28. Eyal T, Steffel M, Epley N. Perspective mistaking: Accurately understanding the mind of another requires getting perspective, not taking perspective. J Pers Soc Psychol. 2018; 114(4): 547-571. http://dx.doi.org/10.1037/pspa0000115

29. Batson CD, Early S, Salvarani G. Perspective taking: Imagining how another feels versus imaging how you would feel. Pers Soc Psychol Bull. 1997; 23(7): 751-758. http://dx.doi.org/10.1177/0146167297237008

30. Bohart AC, Greenberg LS. Empathy reconsidered: New directions in psychotherapy. Washington (DC): American Psychological Association; 1997.

31. Elliott R, Bohart AC, Watson JC, Greenberg LS. Empathy. En: Norcross JC, editor. Psychotherapy relationships that work. 2nd. ed. New York (NY): Oxford University Press; 2011. p. 132-152.

32. Rogers CR. The necessary and sufficient conditions of therapeutic personality change. J Couns Psychol. 1957; 21: 95-103. http://dx.doi.org/10.1037/h0045357

33. Rogers CR. Empathic: An unappreciated way of being. Couns Psychol. 1975; 5(2): 2-10. http://dx.doi.org/10.1177/001100007500500202

34. Stiles WB. Responsiveness as an obstacle for psychotherapy outcome research: It's worse than you think. Clin Psychol-Sci Pr. 2009; 16(1): 86-91. http://dx.doi.org/10.1111/j.1468-2850.2009.01148.x

35. Hatcher RL. Interpersonal competencies: Responsiveness, technique, and training in psychotherapy. Am Psychol. 2015; 70(8): 747-57. http://dx.doi.org/10.1037/a0039803

36. Bloom P. Empathy and its discontents. Trends Cogn Sci. 2017; 21(1): 24-31. http://dx.doi.org/10.1016/j.tics.2016.11.004

37. Bloom P. Against empathy: The case for rational compassion. New York (NY): Ecco; 2016.

38. Khoury B, Sharma M, Rush SE, Fournier C. Mindfulness-based stress reduction for healthy individuals: A meta-analysis. J Psychosom Res. 2015; 78(6): 519-528. http://dx.doi.org/10.1016/j.jpsychores.2015.03.009

39. Galante J, Galante I, Bekkers MJ, Gallacher J. Effect of kindness-based meditation on health and well-being: A systematic review and meta-analysis. J Consult Clin Psychol. 2014; 82(6): 1101-1114. http://dx.doi.org/10.1037/a0037249

40. Bellosta-Batalla M, Ruiz-Robledillo N, Sariñana-González P, Capella-Solano T, Vitoria-Estruch S, HidalgoMoreno $\mathrm{G}$, et al. Increased salivary IgA response as an indicator of immune competence after a mindfulness and self-compassion-based intervention. Mindfulness. 2018; 9(3): 905-913. https://doi.org/10.1007/s12671-017-0830-y 
41. Kabat-Zinn J. Full catastrophe living: Using the wisdom of your body and mind to face stress, pain, and illness. New York (NY): Dell Publishing; 1990.

42. Segal ZV, Williams JMG, Teasdale JD. Mindfulness-based cognitive therapy for depression: A new approach to preventing relapse. New York (NY): Guilford Press; 2002.

43. Goyal M, Singh S, Sibinga EM, Gould NF, Rowland-Seymour A, Sharma R, et al. Meditation programs for psychological stress and well-being: A systematic review and meta-analysis. JAMA Intern Med. 2014; 174(3): 357-368.

http://dx.doi.org/10.1001/jamainternmed.2013.13018

44. Luberto CM, Shinday N, Song R, Philpotts LL, Park ER, Fricchione GL, Yeh GY. A systematic review and meta-analysis of the effects of meditation on empathy, compassion, and prosocial behaviors. Mindfulness. 2018; 9(3): 708-724.

http://dx.doi.org/10.1007/s12671-017-0841-8

45. Donald JN, Sahdra BK, Van Zanden B, Duineveld JJ, Atkins PWB, Marshall SL, Ciarrochi J. Does your mindfulness benefit others? A systematic review and meta-analysis of the link between mindfulness and prosocial behavior. Br J Psychol. 2018.

http://dx.doi.org/10.1111/bjop.12338

46. Hervás G, Cebolla A, Soler J. Intervenciones psicológicas basadas en mindfulness y sus beneficios: Estado actual de la cuestión. Clínica Salud. 2016; 27(3): 115-124.

http://dx.doi.org/10.1016/j.clysa.2016.09.002

47. Hölzel BK, Lazar SW, Gard T, Schuman-Olivier Z, Vago DR, Ott U. How does mindfulness meditation work? Proposing mechanisms of action from a conceptual and neural perspective. Perspect Psychol Sci. 2011; 6(6): 537-559.

http://dx.doi.org/10.1177/1745691611419671

48. Cebolla A, Galiana L, Campos D, Oliver A, Soler J, Demarzo M, et al. How does mindfulness work? Exploring a theoretical model using samples of meditators and non-meditators. Mindfulness. 2018; 9(3): 860-870. http://dx.doi.org/10.1007/s12671-017-0826-7

49. Grossman P. Kindness and compassion as integral to mindfulness. Experiencing the knowable in a special way. En: Singer T, Bolz M, editores. Compassion: Bridging practice and science. Munich: Max Planck Society; 2013. p. 192-207.

50. Bishop SR, Lau M, Shapiro SL, Carlson L, Anderson ND, Carmody J, et al. Mindfulness: A proposed operational definition. Clin Psychol-Sci Pr. 2004; 11(3): 230-241.

http://dx.doi.org/10.1093/clipsy.bph077

51. Brito G, Campos D, Cebolla A. Implicit or explicit compassion? Effects of compassion cultivation training and comparison with mindfulness-based stress reduction. Mindfulness. 2018; 9(5): 1494-1508.

http://dx.doi.org/10.1007/s12671-018-0898-z

52. Kuyken W, Watkins E, Holden E, White K, Taylor RS, Byford S, et al. How does mindfulness-based cognitive therapy work? Behav Res Ther. 2010; 48(11): 1105-1112.

http://dx.doi.org/10.1016/j.brat.2010.08.003

53. Kirby JN, Tellegen CL, Steindl SR. A meta-analysis of compassion-based interventions: Current state of knowledge and future directions. Behav Ther. 2017; 48(6): 778-792.

http://dx.doi.org/10.1016/j.beth.2017.06.003

54. Salzberg S. Real happiness. The power of meditation. NewYork (NY): Workman Publishing; 2011.

55. Neff KD, Germer CK. A pilot study and randomized controlled trial of the mindful self-compassion program. J Clin Psychol. 2013; 69(1): 28-44. http://dx.doi.org/10.1002/jclp.21923

56. Jinpa T. A fearless heart: How the courage to be compassionate can transform our lives. New York (NY): Hudson Street Press; 2015.

57. Strauss C, Taylor BL, Gu J, Kuyken W, Baer R, Jones F, Cavanagh K. What is compassion and how can we measure it? A review of definitions and measures. Clin Psychol Rev. 2016; 47: 15-27. http://dx.doi.org/10.1016/j.cpr.2016.05.004

58. Elices M, Carmona C, Pascual JC, Feliu-Soler A, Martin-Blanco A, Soler J. Compassion and self-compassion: Construct and measurement. Mindfulness Compassion. 2017; 2(1): 34-40. http://dx.doi.org/10.1016/j.mincom.2016.11.003 
59. Coutinho JF, Silva PO, Decety J. Neurosciences, empathy, and healthy interpersonal relationships: Recent findings and implications for counseling psychology. J Couns Psychol. 2014; 61(4): 541-548. http://dx.doi.org/10.1037/cou0000021

60. Preckel K, Kanske P, Singer T. On the interaction of social affect and cognition: Empathy, compassion and theory of mind. Curr Opin Behav Sci. 2018; 19: 1-6. http://dx.doi.org/10.1016/j.cobeha.2017.07.010

61. García-Campayo J, Cebolla A, Demarzo M, editores. La ciencia de la compasión. Más allá del mindfulness. Madrid: Alianza Editorial; 2016.

62. Chierchia G, Singer T. The neuroscience of compassion and empathy and their link to prosocial motivation and behavior. En: Dreher JC, Tremblay L, editores. Decision neuroscience - An integrative perspective. San Diego: Elsevier; 2017. p. 247-257.

63. Siegel RD, Germer CK. Wisdom and compassion in psychotherapy: Deepening mindfulness in clinical practice. New York (NY): Guilford Press; 2012.

64. Shapiro SL, Carlson LE. The art and science of mindfulness: Integrating mindfulness into psychology and the helping professions. Washington (DC): American Psychological Association; 2009.

65. Germer CK, Siegel RD, Fulton PR, editores. Mindfulness and psychotherapy. 2nd. ed. New York (NY): Guilford Press; 2013.

66. Silva-Soler MF, Araya-Véliz C. Influencia de la práctica de mindfulness en la apertura para conocer y comprender a los consultantes en contextos psicoterapéuticos. Un estudio cualitativo desde la perspectiva del terapeuta. Mindfulness Compassion. 2016; 1(2): 84-93.

http://dx.doi.org/10.1016/j.mincom.2016.10.003

67. Feixas G, Miró MT. Aproximaciones a la psicoterapia. Una introducción a los tratamientos psicológicos. Barcelona: Paidós; 1993.

68. Boellinghaus I, Jones FW, Hutton J. Cultivating self-care and compassion in psychological therapists in training: The experience of practicing loving-kindness meditation. Train Educ Prof Psyc. 2013; 7(4): $267-$ 277. http://dx.doi.org/10.1037/a0033092

69. Füstös J, Gramann K, Herbert BM, Pollatos O. On the embodiment of emotion regulation: Interoceptive awareness facilitates reappraisal. Soc Cogn Affect Neurosci. 2013; 8(8): 911-917. http://dx.doi.org/10.1093/scan/nss089

70. Grynberg D, Pollatos O. Perceiving one's body shapes empathy. Physiol Behav. 2015; 140: 54-60. http://dx.doi.org/10.1016/j.physbeh.2014.12.026

71. Bellosta-Batalla M, Pérez-Blasco J, Cebolla A, Moya-Albiol L. Empatía y mindfulness. Convergencia teórica. PsyCap. 2017; 3(1): 34-44

72. Kabat-Zinn J. Wherever you go, there you are: Mindfulness meditation in everyday life. New York (NY): Hyperion; 1994.

(C) 2019 por los autores; Esta obra está sujeta a la licencia de Reconocimiento 4.0 Internacional de Creative Commons. Para ver una copia de esta licencia, visite http://creativecommons.org/licenses/by-nc-nd/4.0/. 